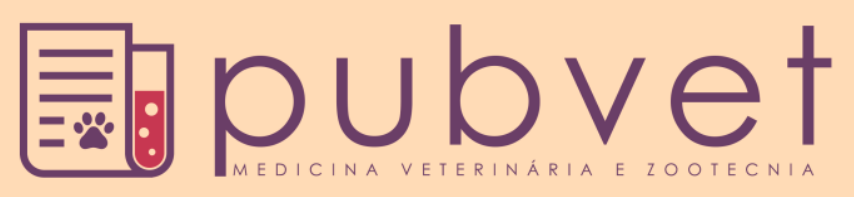

HTTP://DX.DOI.ORG/10.22256/PUBVET.V11N1.91-102

\title{
Avaliação de carcaça ovina por tomografia computadorizada: Estado da Arte no Brasil e no mundo
}

\author{
André Torres Geraldo ${ }^{1^{*}}$, Celia Raquel Quirino ${ }^{{ }^{* *}}$, Renato Travassos Beltrame ${ }^{3}$, Ricardo \\ Lopes Dias da Costa ${ }^{4}$
}

${ }^{1}$ Laboratório de Biotecnologia da Reprodução e Melhoramento Genético Animal, Universidade Estadual do Norte Fluminense/UENF, RJ-Brasil); ** Pesquisadora CNPq

${ }^{2}$ Professor do Centro Universitário do Espírito Santo, Colatina, ES-Brasil

3Pesquisador do Instituto de Zootecnia, Centro de zootecnia diversificada, Nova Odessa, SP-Brasil

*Autor para correspondência: andre_atg@hotmail.com

\begin{abstract}
RESUMO. Várias técnicas são utilizadas para avaliação da carcaça ovina, com objetivo de avaliar os animais destinados ao abate ou seleção de animais com características de deposição e crescimento tecidual que melhor representem o mercado consumidor. Técnicas como avaliação do ganho de peso, do escore corporal e da ultrassonografia de carcaça são largamente utilizadas na rotina de produção; porém são técnicas sujeitas a muitas variações dependendo dos técnicos que as apliquem, e muitas vezes não apresentam resultados tão exatos como outros métodos mais tecnológicos. $\mathrm{O}$ uso da tomografia computadorizada com esse fim ainda está em desenvolvimento no mundo, sendo uma ferramenta altamente precisa para avaliação do crescimento e dos componentes de carcaça de ovinos. Por ser uma análise não invasiva e extremamente acurada, pode apresentar uma importante opção frente aos outros métodos rotineiramente utilizados.
\end{abstract}

Palavras chave: área de olho de lombo, imagem por tomografia, ovinos

\section{Lamb carcass evaluation by computed tomography: State of the art in Brazil and in the world}

\begin{abstract}
Different techniques has been used to evaluate the lamb carcass, aiming to slaughter or animal's selection with deposition and tissue growth traits, most preferred by consumer market. The evaluation of the weight gain, body condition score and carcass ultrasound are widely used in production routine, but this techniques are subjective and to many variations occurs depending on who apply them, often do not show accurate results as other more technology methods. The computed tomography scans (CT) for this purpose is still in progress around the world, being a precise tool for evaluating the growth and sheep carcass components. It is a non-invasive and highly accurate analysis, can provide an important option compared to the other methods routinely used.
\end{abstract}

Keywords: rib eye area, sheep, topographic image

\section{Evaluación de la canal ovina por tomografía computadorizada: Estado del arte en Brasil y en el mundo}

RESUMO. Varias técnicas son utilizadas para la evaluación de la canal ovina, con objetivo de evaluar los animales destinados al sacrificio o selección de animales con características de deposición y crecimiento de tejido que represente mejor el mercado consumidor. Técnicas como evaluación de la ganancia de peso, de clasificación corporal y de 
ultrasonografía de canal son ampliamente utilizadas en la rutina de producción; sin embargo, son técnicas sujetas a muchas variaciones dependiendo de los técnicos que las apliquen, y muchas veces no presentan resultados tan exactos como otros métodos más tecnológicos. El uso de la tomografía computadorizada con ese fin aún está en desarrollo en el mundo, siendo una herramienta altamente precisa para evaluación del crecimiento y de los componentes de la canal ovina. Por ser un análisis no invasiva y extremamente apurada, puede presentar una importante opción frente a los otros métodos de rutina utilizados.

Palabras clave: área del ojo de lomo, imagen por tomografía, ovinos

\section{Introdução}

Nos últimos anos devido ao aumento do mercado consumidor de carne ovina e do maior número de produtores, a ovinocultura se destaca como uma atividade em expansão na maioria dos estados brasileiros (Viana, 2008). De acordo com o IBGE (2013) o Brasil detém de um rebanho de ovinos em torno de 16,789 milhões de animais, podendo o rebanho ovino chegar a 3,0 bilhões em 2050. As maiores participações do efetivo de rebanho ocorreram no Nordeste do País.

A avaliação da carcaça por predição in vivo pode garantir a economicidade do processo produtivo, o que possibilita determinar o grau de terminação e de desenvolvimento muscular dos animais; geralmente essa avaliação é feita por observação visual e palpação (Tarouco et al., 2005).

A ultrassonografia (US) é uma opção interessante para avaliação de carcaças ovinas in vivo, sendo uma técnica relativamente barata $\mathrm{e}$ rápida, que permite avaliar e/ou acompanhar o crescimento do músculo Longissimus dorsi pela através das medidas de comprimento, profundidade e área do músculo (área do olho de lombo), além da avaliação da gordura de cobertura do Longissimus dorsi, com objetivo de detectar o melhor momento para o abate (Suguisawa, 2002). No entanto, a US ainda é uma análise sujeita a erros, dependendo em grande parte da habilidade do técnico e qualidade do equipamento utilizado, o que torna difícil a comparação entre resultados científicos (Bueno et al., 2000).

O uso da tomografia computadorizada (TC) para avaliação de carcaças ainda está em desenvolvimento no mundo, mas é uma ferramenta mais sofisticada e altamente precisa para avaliação do crescimento animal e dos componentes de carcaça de ovinos. Por ser uma análise não invasiva e extremamente acurada (Clelland et al., 2014) pode apresentar uma importante ferramenta para o melhoramento genético de ovinos. O objetivo dessa revisão foi estudar os métodos de avaliação de carcaça para ovinos, com enfoque sobre a tomografia computadorizada.

\section{Características da carcaça ovina}

A carcaça é o elemento mais importante do animal, pois representa a maior porção comestível do corpo, e dessa forma, o objetivo comercial mais rentável. Por esse motivo, suas características devem ser comparadas, de modo que seja possível identificar as diferenças existentes entre os diferentes animais, apontando os superiores. Desse modo, devem-se buscar animais que apresentem carcaças com boa deposição de tecidos comestíveis, o que beneficiará os setores de comercialização (Viana, 2008).

Basicamente os constituintes da carcaça são representados por músculos, ossos e gordura. Cada tecido terá maior desenvolvimento em uma fase diferente da vida do animal. Conforme os animais alcançam a maturidade, ocorrem alterações fisiológicas com relação à deposição de tecidos na carcaça. Como regra geral, a sequência de crescimento dos diferentes tecidos ocorre inicialmente no tecido nervoso, seguido do ósseo, muscular e adiposo (Sainz, 2000). A avaliação dos tecidos da carcaça baseia-se na dissecação dos três principais tipos de tecidos: muscular, adiposo e ósseo (Cezar and Sousa, 2007). O seu conhecimento permite estabelecer um balanço preciso da aptidão do animal, valorizar os tipos genéticos e controlar os sistemas de produção além de detectar as diferenças entre peso vivo, sexo, tipo de nutrição e suas interações (Sen et al., 2004). Na maioria das vezes, o fator que causa maiores problemas sobre a comercialização da carne ovina é a quantidade de gordura. Com o aumento do peso da carcaça pode ocorrer aumento de seu rendimento, no entanto, rendimentos altos podem estar associados a excessivo grau de gordura (Furusho-Garcia et al., 2004) estes altos teores de gordura podem depreciar o valor comercial das carcaças (Alves, 2013). Assim, é necessário certo teor de tecido adiposo como 
determinante das boas características sensoriais da carne e também para reduzir as perdas de água no resfriamento (Silva Sobrinho et al., 2005); porém não em excesso.

Em ovinos, com avanço da idade a deposição de gordura é maior, o que torna importante, portanto considerar o peso de abate, que deve coincidir com o ponto em que a gordura está na proporção desejável, sendo que a duração dessa fase varia, principalmente, de acordo com a raça (Oliveira et al., 2002a).

Quando a terminação dos animais acontece em confinamento, a base de rações com alto teor energético, os animais que possuem característica de maturidade precoce atingem rapidamente à etapa de crescimento, onde maior proporção de energia é depositada como tecido adiposo. Estes animais devem ser abatidos mais precocemente, com objetivo de gerar carcaças com a quantidade de gordura adequada, evitando problemas decorrentes de excesso e, consequentemente, perda de qualidade (Susin and Mendes, 2007). Em um experimento realizado por Santos et al. (2001) com ovinos da raça Santa Inês, avaliou-se o desenvolvimento relativo dos tecido ósseo, muscular e adiposo da carcaça. Os autores concluíram que à medida que aumenta o peso de abate, eleva-se a quantidade de gordura na carcaça e, por intermédio de determinados cortes efetuados é possível verificar, de forma mais acentuada, o aumento de deposição do tecido adiposo.

Azeredo et al. (2005) utilizaram 57 ovinos Corriedale, abatidos em diferentes idades (120, 210 , e 360 dias) com o objetivo de avaliar as características in vivo da carcaça. Os autores concluíram que a idade ao abate influenciou a deposição de gordura na carcaça, e que os animais com 120 dias de idade apresentaram carcaças com maiores proporções de gordura. Assim como a gordura, a dinâmica do crescimento muscular varia de acordo com a fase de desenvolvimento do organismo do animal. Após o nascimento do animal ocorre grande mudança no peso relativo à musculatura, que está sendo influenciada pelas funções do músculo. $\mathrm{Na}$ fase pré-púbere e puberdade, os músculos crescem a uma velocidade uniforme e ocorre grande aumento em tamanho, mas sem grandes transformações no peso relativo. Na fase de terminação existe menos crescimento muscular e maior deposição de gordura (Berg and Butterfield, 1976).
Para a maioria dos mercados consumidores, o cordeiro é a categoria animal que oferece carne a maior aceitabilidade, caracterizada por ser mais macia e rosada, textura lisa, consistência firme e quantidade de gordura adequada. A carne do borrego ainda é macia, mas a cor já é mais forte e avermelhada. As dos animais mais velhos (ovelhas e carneiros) já não são atraentes em virtude de menor maciez, gordura amarelada e sabor mais acentuado (Osório et al., 2009). As fêmeas apresentam carcaças fisiologicamente mais maduras, machos castrados situam-se em uma condição intermediária e os machos não castrados apresentam carcaças mais tardias fisiologicamente. Dessa maneira, a proporção de gordura é maior nas fêmeas, intermediária nos machos castrados e menores nos inteiros, contrariamente à proporção de músculo na carcaça (Cezar and Sousa, 2007). Dessa forma, carcaças de boa qualidade devem apresentar elevada proporção de músculos, baixa proporção de ossos e quantidade adequada de gordura intramuscular para garantir a suculência e o sabor da carne (Eiras et al., 2014).

\section{Métodos de avaliação da carcaça \\ Peso}

$\mathrm{O}$ peso ideal ao abate é aquele onde a proporção de músculos na carcaça é máxima e a gordura é suficiente para conferir propriedades sensoriais adequadas que atendam as preferências do mercado consumidor (Azeredo et al., 2005). Com o incremento do peso corporal ocorre aumento no peso, aumento no rendimento de carcaça, aumento na área de olho de lombo e no teor de gordura, o que melhora a conformação e a compacidade da carcaça. Como aspecto produtivo, deve-se considerar também a eficiência de conversão alimentar do cordeiro, que piora à medida que o peso vivo aumenta (Oliveira et al., $\underline{2002 b)}$.

A meta em ovinos de corte é a obtenção de animais capazes de direcionar grandes quantidades de nutrientes para a produção de músculos, uma vez que o acúmulo desse tecido é desejável e reflete a maior parte da porção comestível de uma carcaça (Santos et al., 2001).

A comercialização em base ao peso vivo e de carcaça, em função do seu rendimento não é a forma mais adequada por não considerar a qualidade do animal como um todo. Para que se determine a qualidade total há necessidade de se estudar os componentes do peso vivo. Dentre 
estes, a carcaça é o de maior valor comercial, mas não é o único (Azeredo et al., 2005).

\section{Condição corporal}

Nos pequenos ruminantes a região lombar sempre foi tida como a melhor região de avaliação da condição corporal (CC). No entanto, devido à escassa cobertura de gordura subcutânea em algumas raças de ovinos, diversas regiões corporais foram testadas para o exame da CC, entre estas a base da cauda em ovinos.

A condição corporal é amplamente utilizada para avaliar o grau de terminação do animal, permitindo um entendimento com relação à deposição de gordura da carcaça devido ao fato de servir como indicativo da quantidade de gordura subcutânea. Assim, o conhecimento da condição corporal e das características in vivo é importante para efetuar a avaliação dos animais, já que essas características podem variar segundo genótipo, sexo, idade e peso corporal (Sañudo et al., 2004). Jefferies (1961) foi o primeiro pesquisador a utilizar o método de avaliação da CC, através de uma escala pela palpação de 5 pontos (0-muito magro, 5-muito gordo) com a determinação do grau de cobertura tissular da região lombar das ovelhas. A correlação entre a CC e a deposição de gordura na carcaça é de média a alta, entretanto os resultados entre os coeficientes de correlação ainda podem variar muito entre as pesquisas (Osório et al., 2009).

Em trabalho realizado no Brasil, Cartaxo and Sousa (2008) avaliaram a viabilidade de produção de cordeiros Santa Inês e Dorper abatidos em diferentes escalas de $\mathrm{CC}$ e observaram que os animais abatidos em escore intermediário (2,5 a $3,5)$ apresentam menor consumo de matéria seca, melhor conversão alimentar, menor número de dias em confinamento. Bonacina et al. (2007) avaliaram a correlação entre a CC e o estado de acabamento de carcaça. Os autores observaram que existe correlação $(0,43$ avaliador 1 e 0,52 avaliador 2) entre as notas de escore corporal (15 ) e as notas para cobertura de gordura da carcaça (1-5), ambas subjetivas.

Cartaxo et al. (2011) avaliaram o desempenho e as características qualitativas de cordeiros (Santa Inês, Santa Inês $\mathrm{x}$ Dorper) terminados em confinamento. Pelos resultados foi possível observar que os cordeiros mestiços apresentaram melhor conformação e maior espessura de gordura subcutânea. A medição realizada entre a $12^{\mathrm{a}}$ e $13^{\mathrm{a}}$ costelas é aceita como bom indicador, representando a composição tecidual do corpo inteiro do animal (Luchiari Filho, 2000).

Sá \& Otto de Sá (2001) classificaram o CC de ovinos em uma escala de 5 pontos, sendo EC1 muito magra, e sem gordura de cobertura, até EC5, muito gorda e com excesso de gordura (Figura 1).

\section{Ultrassonografia}

O aparelho de ultrassom basicamente mede a reflexão das ondas de alta frequência que ocorre quando estas passam pelos tecidos. Após o transdutor ter sido colocado, ocorre a conversão de pulsos elétricos em ondas de alta frequência (ultrassons), que ao encontrar diferentes densidades dentro do animal promove uma reflexão parcial (eco), em tecidos de menor densidade ou total em tecidos de maior densidade. Após a ocorrência do eco, as ondas de alta frequência continuam a se propagar pelo corpo do animal e as informações enviadas pelas reflexões transmitidas ao transdutor são convertidas em imagem (Suguisawa, 2002).

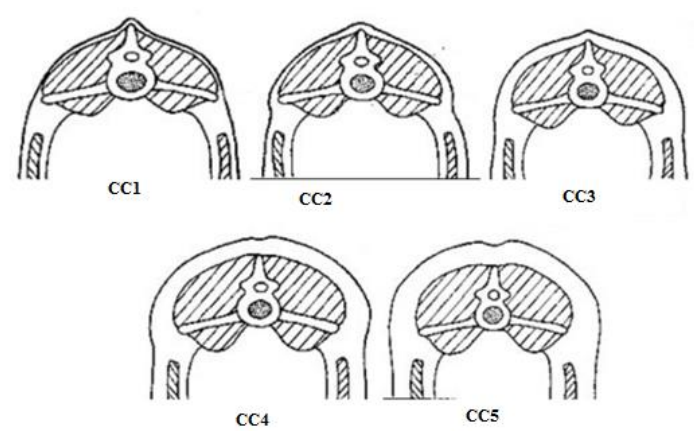

Figura 1. Avaliação de Escore Corporal (CC). (Adaptado de Sá \& Otto de Sá, 2000).

O uso da ultrassonografia para avaliação de carcaça é uma técnica eficaz para avaliação das características produtivas e reprodutivas (Silva et al., 2005) sendo utilizada a campo com aparelhos portáteis, mostrando alta repetibilidade, baixo custo e fácil operação, sendo rápida e objetiva alcançando as demandas do mercado sem danos ao produto final (Ripoll et al., 2010).

Em ovinos, assim como em bovinos, são medidas a área de olho de lombo, comprimento e profundidade do músculo e espessura de gordura subcutânea (EG) na secção do músculo Logissimus dorsi, a partir de imagens tomadas entre a 12 $2^{\mathrm{a}}$ e $13^{\mathrm{a}}$ costelas (Figura 2) (Duran, 2012).

A área do músculo Longissimus dorsi, comumente chamada área olho de lombo, apresenta correlação positiva com a quantidade de carne vendável da 
carcaça, enquanto que a espessura da camada de gordura subcutânea (EG) correlaciona-se positivamente com a quantidade total de gordura acumulada no corpo do animal (Pérez and Carvalho, 2002). Embora a US já esteja consolidada para o meio cientifico, alguns trabalhos ainda apresentam resultados discrepantes em relação às mesmas medidas feitas na carcaça, fato que provavelmente ocorre pelas mensurações a serem realizadas em posições diferentes, o que compromete muito as comparações feitas entre as mesmas (Suguisawa, 2002).

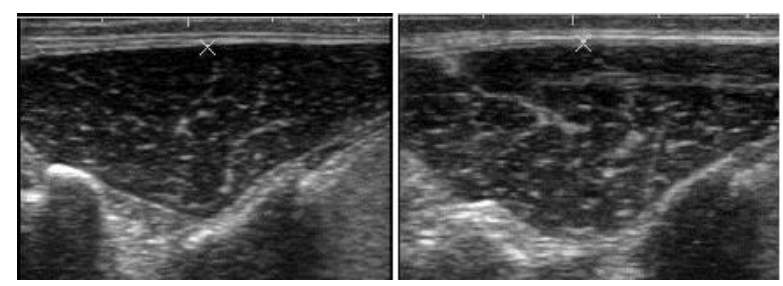

Figura 2. Imagem ultrassonográfica da área de olho de lombo de ovinos (Arquivo pessoal)

\section{Dissecação}

O método que pode ser considerado mais preciso para a estimativa da composição tecidual é a dissecação de toda a carcaça em três principais grupos de tecidos (ósseo, muscular e adiposo) e a determinação de suas proporções (Geraldo et al., 2015). Todavia, a dissecação de apenas um dos lados ou de um corte representativo da carcaça também pode ser utilizada com sucesso para essa determinação (Cezar and Sousa, 2007). A paleta e a perna são os cortes mais utilizados para predizer a composição tecidual da carcaça, sendo considerados indicadores confiáveis da sua proporção tecidual. No entanto, Silva et al. (2000) avaliaram características de carcaça de cordeiros abatidos com diferentes idades e suas correlações com as proporções de tecidos através da dissecação de vários cortes, constatando que a costela é o corte que melhor expressa a proporções de osso, músculo e gordura da carcaça. Apesar das vantagens conferidas pela técnica de dissecação, esta é considerada onerosa e demorada, além de exigir geralmente o descarte das carnes provenientes dos animais dissecados. Além disso, as diferenças no treinamento dos técnicos que realizam a análise levam a uma grande variação dos resultados obtidos (Cezar and Sousa, 2007).

\section{Tomografia computadorizada}

O corte transversal em três locais identificados a partir da varredura topográfica $\left(5^{\mathrm{a}}\right.$ vértebra lombar (VL5), $8^{\mathrm{a}}$ vértebra torácica (VT8) e ísquio (ISQ) (Figura 3) fornecem imagens adequadas para medições da área muscular, gordura e ossos, além de prever com boa precisão o peso dos componentes (Anderson et al., 2015). Este modo de análise foi desenvolvido com intenção de maximizar a precisão da leitura além de proceder à análise no menor tempo possível, para minimizar as implicações ao bem estar dos animais (Bünger et al., 2011).

A TC é uma tecnologia sofisticada, que pode oferecer informações objetivas e confiáveis. Está análise é feita através do uso de raios-x que geram imagens bidimensionais transversais do corpo, obtidas pela rotação de um tubo de raio-x de $360^{\circ}$ em torno do corpo do animal. Após a digitalização da imagem, o objeto (corpo) é dividido em várias seções consecutivas, paralelas e os dados são somados para produzir estimativas totais dos diferentes tecidos da carcaça (Bünger et al., 2011). As imagens geradas são descritas em vários tons de cinza, criando uma imagem espacial do objeto digitalizado (Wegener, 1992).

A tomografia computadorizada é utilizada na clínica médica como forma de diagnóstico desde 1970; a partir de 1980 a tecnologia começou a ser utilizada na Europa como forma de predição da composição de carcaça e crescimento animal (Standal, 1984). No início, os trabalhos foram realizados com suínos, em seguida com ovinos, em carcaças e animais vivos (Sonesson et al., 1998, Jones et al., 1999). Devido a fatores como pouco acesso ao equipamento e alto custo de execução, a TC não foi uma tecnologia amplamente difundida para predição de carcaças (Rivero et al., 2005). Os resultados mostraram altas correlações entre as proporções dos tecidos de carcaça, estimadas por TC, quando comparadas à dissecação realizada por técnicos treinados (Sehested, 1986) com menor erro padrão com relação às estimativas realizadas por ultrassonografia, técnica amplamente utilizada na predição de tecido de carcaça até hoje (Anderson et al., 2015).

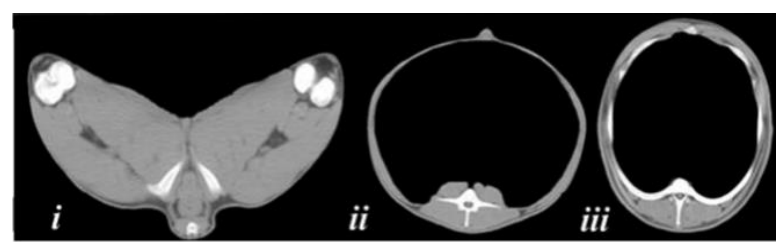

Figura 3. Cortes longitudinais de ovinos por tomografia computadorizada. $i$ - Ísquio, $i i-5^{\mathrm{a}}$ Vértebra Lombar, iii $-8^{\mathrm{a}}$ vértebra torácica. Adaptado de Clelland et al. (2014). 
Com a maior disponibilidade do equipamento e evolução do uso da técnica, a TC já tem uma vasta gama de uso em animais, em especial nas análises de animais vivos, para fins de reprodução e avaliação de carcaça post-mortem, processos que já são considerados de rotina em alguns países (Bünger et al., 2011).

Vários estudos foram realizados com a utilização da TC em ovinos e suínos no mundo, tendo a dissecação manual como referência, (Dobrowolski et al., 2004, Jones et al., 2002, Johansen et al., 2007, Navajas et al., 2007). No entanto, existem diferenças nos resultados da técnica de referência da dissecação entre os técnicos utilizados nos experimentos (Nissen et al., 2006).

A técnica foi estuda em vários países, sendo que a predição de composição de carcaça de ovinos usando TC foi incluída nos programas de reprodução e melhoramento genético do Reino Unido, Noruega e Nova Zelândia (Nicoll et al., 2002, Kvame et al., 2006, Macfarlane et al., 2006).

Uma abordagem alternativa na medição dos tecidos por TC é o Método Cavalieri, que utiliza 15 a 20 imagens transversais contínuas uniformemente distribuídas ao longo da carcaça, desse modo, o volume de cada tecido é calculado a partir da área total, (a partir do corte transversal multiplicado pela distância entre as verificações) (Roberts et al., 1993). Ambos os métodos mostram precisões similares em ovelhas, mas exigem tempos muito diferentes (economicamente importante) para geração das imagens e no tempo de análise. Dessa forma o método de varredura topográfica utilizando os 3 locais de referencia (VL5, VT8 e ISQ) é geralmente preferido (Jones et al., 2004).

\section{Predição de gordura pela TC}

A gordura intramuscular (marmoreio) tem um papel de extrema importância do ponto de vista de qualidade de carne, pois influencia em larga escala características organolépticas do produto, principalmente a textura e sabor. Desse modo, a predição dessa gordura na carcaça de ovinos é de extrema importância para a cadeia produtiva, pois permite a classificação do produto e seu direcionamento para diferentes mercados, que procuram por qualidade diferenciada do produto (Bueno et al., 2000).

A análise da gordura intramuscular pode ser realizada pela US, geralmente pela de uma análise subjetiva, pontuando de 1 (ausência de marmoreio) a 5 (excesso de marmoreio) as imagens ultrassonográficas dos músculos de animais vivos. O músculo mais utilizado nesse tipo e análise é o Longissimus dorsi, na região intervertebral entre a $12^{\circ}$ e $13^{\circ}$ costelas (Suguisawa, 2002). No entanto, a tomografia computadorizada pode ser utilizada com maior precisão que a US, identificando a quantidade total de gordura $(\mathrm{kg})$ através da escala Houndsfield (Clelland et al., 2014).

Em um estudo desenvolvido por Clelland et al. (2014) utilizando a TC para predição de gordura intramuscular em ovinos da raça Texel, com imagens nas regiões da VL5, VT8 e ISQ, coletadas em diferentes anos, analisadas através do software STAR para ovinos. Os autores concluíram com base nos resultados obtidos entre a análise tomográfica e a dissecação, que a tomografia computadorizada é um bom método na predição de gordura intramuscular em ovinos.

A gordura interna também é de grande importância para a produção animal, muitas espécies possuem grande quantidade dessa gordura em sua carcaça, podendo trazer várias consequências negativas para a produção de forma geral (Bueno et al., 2000). O acúmulo de gordura exige grande gasto enérgico, o que diminui a eficiência alimentar dos animais, pois existe grande demanda energética para a deposição de gordura e baixa demanda na deposição de tecido muscular. Além disso, quanto maior a quantidade de gordura interna, menores serão os rendimentos de carcaça obtidos. Na maioria das vezes, a gordura interna não é utilizada para alimentação e nem pela indústria, e dessa forma, acaba sendo descartada (Bueno et al., 2000). Nesse sentido, a tomografia computadorizada pode ser utilizada para a medição das quantidades de gordura em animais vivos, podendo contribuir na seleção de animais que tenham como característica baixa deposição de gordura abdominal.

Lambe et al. (2006) mediram a quantidade de gordura interna pela tomografia computadorizada em 427 cordeiros Scottish Blackface, com objetivo de estimar a correlação entre os diferentes tecidos da carcaça e comparar os valores obtidos com os resultados da dissecação manual. Para o estudo foram tomadas 5 diferentes medidas, sendo as imagens retiradas da área do ísquio, perna, VL5, VL2 e VT8. Como resultados a gordura interna apresentou correlação positiva com a gordura de carcaça $(0,58)$, com tecido muscular $(0,36)$ e com os ossos $(0,32)$. A correlação entre os valores 
obtidos pela tomografia computadorizada e dissecação manual foi de 0,81 , mostrando que a tecnologia tem grande potencial para ser utilizada na medição de gordura interna de animais vivos (Figura 4).

Tomografia computadorizada para predição de composição corporal em cordeiros no mundo

O entendimento da composição tecidual é de grande importância, não só para ciência, mas também para o setor industrial. Muitas vezes a análise de composição em ovinos é realizada através da dissecação manual, que além de ser um método caro, também é demorado. No entanto a TC representa uma alternativa para realização da predição dos componentes corporais (Jones et al., 2004).

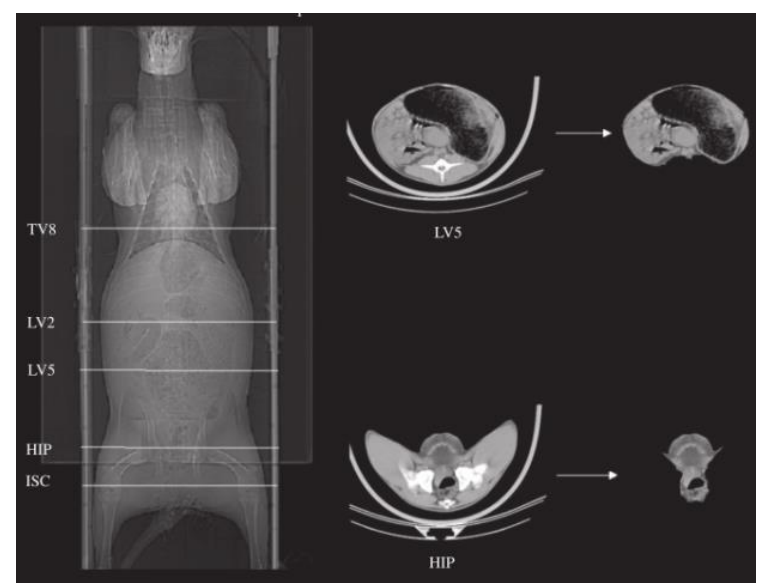

Figura 4. Cortes utilizados para mensuração da gordura interna (Lambe et al., 2006).

Junkuszew and Ringdorfer (2005) avaliaram a predição da composição tecidual em ovinos através da tomografia computadorizada e ultrassonografia. Foram utilizados 41 cordeiros machos (19 Merinos, 14 Suffolk e 8 Texel). Após as análises os animais foram abatidos e a meia carcaça direita foi dissecada. A quantidade total de gordura na carcaça dos animais variou de 8,8 a $28,5 \%$, enquanto a quantidade de músculos variou de 49,5 a $67,4 \%$. As correlações entre a dissecação para gordura foram de 0,88 e 0,72 para tomografia e ultrassonografia, respectivamente, e para os músculos as correlações foram de 0,68 e 0,48 para tomografia e ultrassonografia, respectivamente. Os autores concluíram que ambas as técnicas de predição são eficazes, sendo que a tomografia é um método mais acurado, em contraponto, a ultrassonografia é mais barata e com maior disponibilidade de utilização a campo.

Kongsro et al. (2008) realizaram um trabalho na Noruega, onde avaliaram as correlações entre as proporções dos tecidos de carcaça obtidos pela tomografia computadoriza e por dissecação manual, além da avaliação do escaneamento em diferentes distancias entre os cortes $(40,80,160 \mathrm{e}$ $320 \mathrm{~mm}$ ). As carcaças foram escaneadas e dissecadas manualmente por técnicos treinados. As correlações entre as duas técnicas foram maiores para peso de carcaça e peso de tecidos musculares e menores para ossos e gordura. Os autores concluíram que a melhor distância para estimar os tecidos de carcaça foi de $40 \mathrm{~mm}$.

\section{Comprimento de coluna vertebral e fêmur}

Navajas et al. (2006) pelo método de avaliação proposto por Jones et al. (2002) utilizaram a TC para medição do fêmur e da coluna vertebral, em associação com a musculosidade destas regiões em ovinos Texel e Scottish Blackface. Para a análise de comprimento da coluna foram medidas separadamente (pelas imagens topográficas) a coluna torácica (primeiro disco intervertebral até a última costela) e a coluna lombar (primeiro disco caudal até a última costela). Para medição de fêmur foi utilizado um novo método, que permite a visualização do fêmur em 3 diferentes planos simultaneamente, permitindo a visão longitudinal, lateral e transversal. O comprimento do fêmur foi medido do intercôndilo à fossa trocantérica (Figura 5). Os resultados mostraram que as medidas do fêmur e da coluna obtiveram moderadas e altas precisões quando comparadas as medidas realizadas na carcaça após o abate.

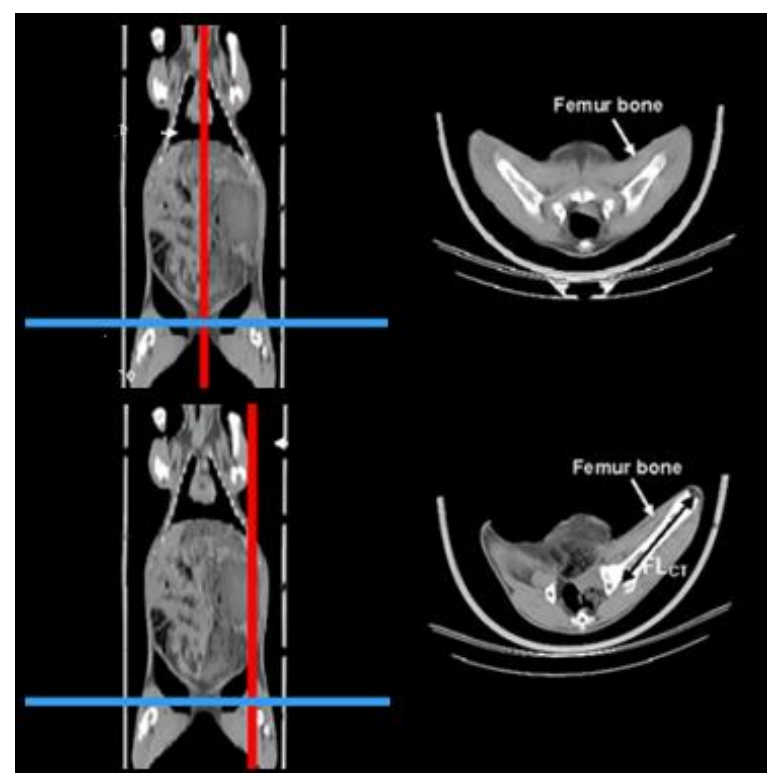

Figura 5. Medição lateral e longitudinal do fêmur por tomografia. Adaptado de Navajas et al. (2006). 
Comparações entre raças e linhagens genéticas utilizando TC

Kvame and Vangen (2006) avaliaram através da tomografia computadorizada a carcaça de ovinos de duas linhagens genéticas da raça Norwegian White Sheep, uma melhorada geneticamente para produção de carne e outra controle. Os pesquisadores constataram que os animais melhorados possuíam maiores proporções de músculo nas regiões analisadas, além de menor proporção de ossos, mostrando a eficiência da tomografia na identificação de animais com melhores características de carcaça.

Lambe et al. (2006) pesquisaram as mudanças corporais em função do crescimento em cordeiros das raças Texel e Scottish Blackface. Através das medidas tomográficas os autores concluíram que os cordeiros Texel acumulam pouca quantidade de gordura e grande quantidade muscular na região da perna e lombo, podendo dessa forma ser abatidos mais pesados. No entanto os cordeiros Scottish Blackface acumulam gordura muito precocemente, e acaba tendo pouco crescimento muscular, o que sugere que esses animais teriam melhor aproveitamento de carcaça sendo sujeitos ao abate precoce.

\section{Tomografia computadorizada no Brasil}

O grupo de pesquisa do Laboratório de Melhoramento Genético Animal (LRMGA) da Universidade Estadual do Norte Fluminense (UENF) vem trabalhando desde 2013 com avaliação de carcaça ovina através da tomografia computadorizada. O tomógrafo utilizado é um LighsSpeed Helicoidal Multslice GE® localizado na Unidade de Experimentação Animal (UEA), do Hospital veterinário da UENF (Figura 6).

Geraldo et al. (2015) compararam medidas do músculo Longissimus dorsi obtidas por tomografia computadorizada e ultrassonografia, na região entre a $12^{\mathrm{a}}$ e $13^{\mathrm{a}}$ costelas de machos. Os autores não encontraram diferenças entre as avaliações pelas duas técnicas nas medições de profundidade do músculo e espessura de gordura subcutânea, mas houve diferença nas medidas do comprimento.
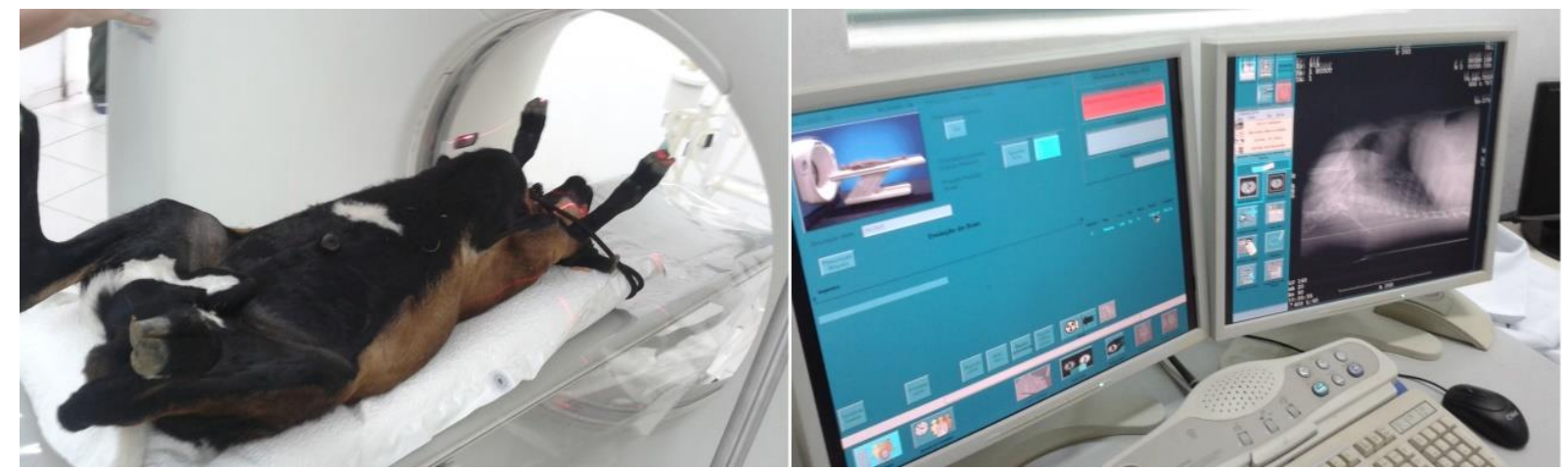

Figura 6. Tomógrafo LighsSpeed Helicoidal Multslice GE ${ }^{\circledR}$ - Universidade Estadual do Norte Flunimense.

Quirino et al. (2016) avaliaram as características de carcaça de ovinos da raça Santa Inês machos e fêmeas pela tomográfica computadorizada, em diferentes regiões anatômicas. Foram utilizados machos $(n=8)$ e fêmeas $(n=7)$ da raça Santa Inês com 150 dias de idade.

Antes do exame tomográfico foi realizado jejum sólido de 14 horas.

Para o exame tomográfico os animais foram submetidos à sedação leve com utilização de cetamina e xilazina, acomodados em maca (decúbito dorsal) e escaneados tomógrafo LighsSpeed Helicoidal Multslice GE® foram retiradas imagens de cortes transversais do músculo Longissimus dorsi correspondentes a quinta vértebra torácica (VT5), oitava vértebra lombar (VL8), décima terceira vértebra lombar (VL13) e uma imagem do ísquio (ISQ).

As imagens foram processadas e analisadas com o software K-pacs $®$, sendo realizadas as medidas de comprimento (Comp), profundidade (Prof) e espessura de gordura subcutânea (EGS) no músculo Longissimus dorsi, e para o corte do ísquio as medidas de comprimento e espessura muscular $(\mathrm{cm})$, além de espessura de gordura intermuscular $(\mathrm{mm})$.

Os autores verificaram que não houve diferença entre sexos para Comp $(6,4 \pm 0,5 \mathrm{~cm})$, Prof $(2,1 \pm 0,4 \mathrm{~cm})$ e EGS $(1,2 \pm 0,4 \mathrm{~mm})$ no corte de VT5. No corte VL8 as medidas de Comp e de EGS foram diferentes entre sexos, sendo 
superiores nos machos $(4,67 \pm 0,22 \mathrm{~cm}$ e $3,27 \pm$ $0,77 \mathrm{~mm})$ em relação as fêmeas $(4,26 \pm 0,33 \mathrm{~cm}$ e $1,71 \pm 0,71 \mathrm{~mm})$. Nesta região não houve diferença entre sexo em Prof $(2 \pm 0,3 \mathrm{~cm})$.

O corte transversal tomado na VL13 não apresentou diferença entre os sexos para as medidas, sendo para Comp 5,6 $\pm 0,5 \mathrm{~cm}$, para Prof $2,6 \pm 0,3 \mathrm{~cm}$ e para EGS $1,7 \pm 0,5 \mathrm{~mm}$.

O corte do ISQ também não apresentou diferenças entre os sexos e as médias foram de $14,8 \pm 2,4 \mathrm{~cm}$ para Comp, de 7,91 $\pm 1,3 \mathrm{~cm}$ para Prof e de $5 \pm 33 \mathrm{~mm}$ para EGS.

$\mathrm{O}$ peso dos animais também não foi diferente entre os sexos (média de $28 \pm 4,4 \mathrm{~kg}$ ). As correlações foram baixas entre as medidas VT5, VL8, VL13 e ISQ. Os autores concluíram que, das regiões anatômicas avaliadas, a medida na VL8 foi a mais eficiente para detectar as variações do comprimento do músculo Longissimus dorsi e deposição de gordura decorrentes do sexo.Geraldo et al. (2016) avaliaram características de carcaça de ovinos da raça Santa Inês machos e fêmeas através de ultrassonografia (US) e tomográfica computadorizada (TC), além da correlação entre os dois métodos (Figura 7). Foram utilizados 17 cordeiros da raça Santa Inês ( 9 machos e 8 fêmeas) com idade de 5 meses $(5 \mathrm{M})$, e 7 machos adultos com 22 meses (22M). As medidas foram realizadas no músculo Longissimus dorsi, na região entre a $12^{\mathrm{a}}$ e $13^{\mathrm{a}}$ costela, onde foram tomadas as medidas de comprimento, profundidade e espessura de gordura subcutânea.

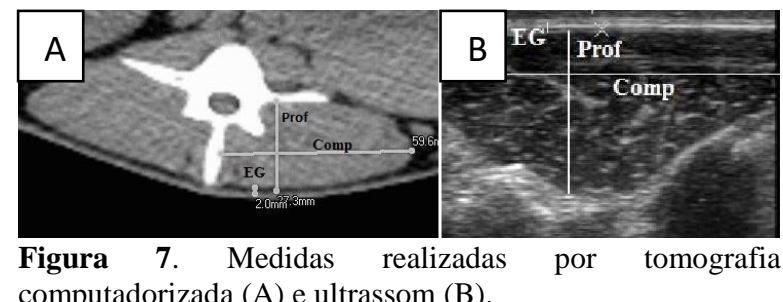

Foi encontrada diferença entre sexos, sendo de $5,0 \pm 0,7 \mathrm{~cm}$ para comprimento, $2,5 \pm 0,6 \mathrm{~cm}$ para profundidade e 2,0 $\pm 0,5 \mathrm{~mm}$ para espessura de gordura nos machos. Nas fêmeas as medias foram de 4,4 $\pm 0,5 \mathrm{~cm}$ para comprimento, $2,1 \pm 0,3 \mathrm{~cm}$ para profundidade e 1,8 $\pm 0,4$ espessura de gordura. Nas medidas tomográficas, a única que apresentou diferença com relação ao sexo foi comprimento do músculo, sendo maior em machos $(6,1 \pm 0,6 \mathrm{~cm})$ em relação às fêmeas $(5,4$ $\pm 0,6 \mathrm{~cm})$. Todas as características foram superiores para os animais do grupo de $22 \mathrm{M}$ de idade. As correlações obtidas entre as medidas ultrassonográficas e tomográficas foram médias e altas, variando entre 0,35 a 0,88 . Os autores concluíram que as médias e altas correlações entre as medidas indicam que ambas as técnicas são semelhantes para as avaliações do músculo Longisimus dorsi em ovinos, no entanto, a ultrassonografia tem maior potencial para uso a campo, sendo mais barata e rápida.

\section{Considerações finais}

A tomografia computadorizada é uma importante ferramenta, que pode ser utilizada para avaliação de diversos constituintes de caraça dos ovinos, com grande potencial para ser utilizada em programas de melhoramento genético, e na indústria animal para obtenção de um produto de melhor qualidade, podendo atingir mercados mais exigentes. Entretanto, visto a alta correlação que apresenta com as medidas tomadas por US, podese concluir que o uso do US seria mais prático e econômico para realizar avaliações de animais a campo.

\section{Referências Bibliográficas}

Alves, L. G. C. 2013. Composição regional e tecidual de cordeiros terminados com dietas contendo grão de soja in natura ou desatvado. Universisdade Federal da Grande Dourados.

Anderson, F., Williams, A., Pannier, L., Pethick, D. W. \& Gardner, G. E. 2015. Sire carcass breeding values affect body composition in lambs-1. Effects on lean weight and its distribution within the carcass as measured by computed tomography. Meat science, 108, 145-154.

Azeredo, D. M., Osório, M. T. M., Osório, J. C. S., Mendonça, G., Barbosa, J. \& Esteves, R. 2005. Crescimento e desenvolvimento de ovinos Corriedale não castrados, castrados e criptorquidas abatidos com diferentes pesos. Revosta Brasileira de Agrociências, 11, 339345.

Berg, R. T. \& Butterfield, R. M. 1976. New concepts of cattle growth. Sydney University Press, Sydney.

Bonacina, M., Osório, J. C. d. S., Osório, M. T. M., Esteves, R. M. G., Jardim, R. D., Mendonça, G. \& Oliveira, M. M. 2007. Otimização da avaliação in vivo e da carcaça em cordeiros. Revista da Faculdade de Zootecnia, Veterinária e Agronomia, 14, 273286. 
Bueno, M. S., Cunha, E., Santos, L., Roda, D. S. \& Leinz, F. F. 2000. Características de carcaça de cordeiros Suffolk abatidos em diferentes idades. Revista Brasileira de Zootecnia, 29, 1803-1810.

Bünger, L., Glasbey, C. A., Simm, G., Conington, J., Macfarlane, J. M., McLean, K. A., Moore, K. \& Lambe, N. R. 2011. Use of X-ray computed tomography (CT) in UK sheep production and breeding. CT ScanningTechniques Applied, 1, 329-348.

Cartaxo, F. Q., Sousa, W., Costa, R. G., Cezar, M. F., Pereira Filho, J. \& Cunha, M. d. G. G. 2011. Características quantitativas da carcaça de cordeiros de diferentes genótipos submetidos a duas dietas. Revista Brasileira de Zootecnia, 40, 2220-2227.

Cartaxo, F. Q. \& Sousa, W. H. 2008. Correlações entre as características obtidas in vivo por ultra-som e as obtidas na carcaça de cordeiros terminados em confinamento. Revista Brasileira de Zootecnia, 37, 1490-1495.

Cezar, M. \& Sousa, W. 2007. Carcaças ovinas e caprinas: obtenção, avaliação e classificação. Uberaba: Editora Agropecuária Tropical, 147.

Clelland, N., Bunger, L., McLean, K. A., Conington, J., Maltin, C., Knott, S. \& Lambe, N. R. 2014. Prediction of intramuscular fat levels in Texel lamb loins using X-ray computed tomography scanning. Meat Science, 98, 263-271.

Dobrowolski, A., Branscheid, W., Romvari, R., Horn, P. \& Allen, P. 2004. X-ray computed tomography as possible reference for the pig carcass evaluation. Fleischwirtschaft, 84, 109112.

Duran, R. R. 2012. Rendimiento en canal de ovinos de pelo al ser alimentados con fruto de guazama uimifolia en sustitución de gano de maiz.

Eiras, C. E., Araújo Marques, J., Novais, D. L., Mottin, C., Maggioni, D. \& Prado, I. N. 2014. Ultrassonografia na avaliação da composição corporal de ruminantes: Revisão. Campo Digital, 9, 110-116.

Furusho-Garcia, I. F., Perez, J. R. O., Bonagurio, S., Lima, A. L. \& Quintão, F. A. 2004. Estudo dos cortes da carcaça de cordeiros Santa Inês puros e cruzas Santa Inês com Texel, Ile de France e Bergamácia. Revista Brasileira de Zootecnia, 33, 453-462.
Geraldo, A. T., Quirino, C. R., Antunes, F., Mendes, L. R. \& Oliveira, A. L. A. 2015. Comparion of the ultrasound and computed tomography to evaluate the Longissimus dorsi muscle in rams. Boletim da Indústria Animal, 1 , Anais.

Geraldo, A. T., Quirino, C. R., Beltrame, R. T., Costa, R. L. D., Antunes, F., Freitas, A. C. B. \& Vieira, G. S. 2016. Avaliação das caratcerísticas de carcaça de ovinos Santa Inês com auxílio da ultrassonografia e tomografia computadorizada. Actas Iberoamericanas, 7, 37-43.

IBGE. 2013. Contas nacionais trimestrais. Jul.-set. 2013. 20th ed. Available in: www.ibge.gov.br, Rio de Janeiro, BR.

Jefferies, B. C. 1961. Body condition scoring and its use in management. Tasmanian Journal of Agriculture, 32, 19-21.

Johansen, J., Egelandsdal, B., Røe, M., Kvaal, K. \& Aastveit, A. H. 2007. Calibration models for lamb carcass composition analysis using Computerized Tomography (CT) imaging. Chemometrics and Intelligent Laboratory Systems, 87, 303-311.

Jones, H. E., Lewis, R. M., Young, M. J. \& Simm, G. 2004. Genetic parameters for carcass composition and muscularity in sheep measured by X-ray computer tomography, ultrasound and dissection. Livestock Production Science, 90, 167-179.

Jones, H. E., Lewis, R. M., Young, M. J. \& Wolf, B. T. 2002. The use of X-ray computer tomography for measuring the muscularity of live sheep. Animal Science, 75, 387-399.

Jones, H. E., Simm, G., Dingwall, W. S. \& Lewis, R. M. 1999. Genetic relationships between visual and objective measures of carcass composition in crossbred lambs. Animal Science, 69, 553-561.

Junkuszew, A. \& Ringdorfer, F. 2005. Computer tomography and ultrasound measurement as methods for the prediction of the body composition of lambs. Small Ruminant Research, 56, 121-125.

Kongsro, J., Røe, M., Aastveit, A. H., Kvaal, K. \& Egelandsdal, B. 2008. Virtual dissection of lamb carcasses using computer tomography (CT) and its correlation to manual dissection. Journal of Food Engineering, 88, 86-93.

Kvame, T., Brenøe, U. T. \& Vangen, O. 2006. Body tissue development in lambs of two 
genetic lines analysed by X-ray computer tomography. Small Ruminant Research, 65, 242-250.

Kvame, T. \& Vangen, O. 2006. In-vivo composition of carcass regions in lambs of two genetic lines, and selection of CT positions for estimation of each region. Small Ruminant Research, 66, 201-208.

Lambe, N. R., Conington, J., McLean, K. A., Navajas, E. A., Fisher, A. V. \& Bünger, L. 2006. In vivo prediction of internal fat weight in Scottish Blackface lambs, using computer tomography. Journal of Animal Breeding and Genetics, 123, 105-113.

Luchiari Filho, A. 2000. Pecuária da carne bovina, 1 edn. LinBife, São Paulo.

Macfarlane, J. M., Lewis, R. M., Emmans, G. C., Young, M. J. \& Simm, G. 2006. Predicting carcass composition of terminal sire sheep using X-ray computed tomography. Animal Science, 82, 289-300.

Navajas, E. A., Glasbey, C. A., McLean, K. A., Fisher, A. V., Charteris, A. J. L., Lambe, N. R., Bünger, L. \& Simm, G. 2006. In vivo measurements of muscle volume by automatic image analysis of spiral computed tomography scans. Animal Science, 82, 545-553.

Navajas, E. A., Lambe, N. R., McLean, K. A., Glasbey, C. A., Fisher, A. V., Charteris, A. J. L., Bünger, L. \& Simm, G. 2007. Accuracy of in vivo muscularity indices measured by computed tomography and their association with carcass quality in lambs. Meat Science, 75, 533-542.

Nicoll, G. B., Jopson, N. B. \& McEwan, J. C. 2002. Contribution of CT scanning to genetic improvement in a terminal sire sheep breeding programme. Genetic Applied Livestock Production, 31, 439-442.

Nissen, P. M., Busk, H., Oksama, M., Seynaeve, M., Gispert, M., Walstra, P., Hansson, I. \& Olsen, E. 2006. The estimated accuracy of the EU reference dissection method for pig carcass classification. Meat Science, 73, 22-28.

Oliveira, M. V. M., Pérez, J. R. O., Alves, E. L., Martins, A. R. V. \& Lana, R. 2002a. Avaliação da composição de cortes comerciais, componentes corporais e órgãos internos de cordeiros confinados e alimentados com dejetos de suínos. Revista Brasileira de Zootecnia, 31, 1459-1468.
Oliveira, M. V. M., Pérez, J. R. O., Alves, E. L., Martins, A. R. V. \& Lana, R. d. P. 2002b. Rendimento de carcaça, mensurações e peso de cortes comerciais de cordeiros Santa Inês e Bergamácia alimentados com dejetos de suínos em confinamento. Revista Brasileira de Zootecnia, 31, 1451-1458.

Osório, J. C. S., Osório, M. T. M. \& Sañudo, C. 2009. Características sensoriais da carne ovina. Revista Brasileira de Zootecnia, 38, 292-300.

Pérez, J. R. O. \& Carvalho, P. A. 2002. Considerações sobre carcaças ovinas. Boletim Agropecuário,, 1, 122-144.

Quirino, C. R., Geraldo, A. T., Antunes, F., Vieira, G. S., Rua, M. A. S., Freitas, A. C. B., David, C. M. \& Bartholazzi Júnior, A. 2016. Avaliação de carcaça de ovinos através da tomografia computadorizada. In: ALPA (ed.) XXV Congresso de la Associación Latinoamericana de Produción Animal. ALPA, Recife.

Ripoll, G., Joy, M. \& Sanz, A. 2010. Estimation of carcass composition by ultrasound measurements in 4 anatomical locations of 3 commercial categories of lamb. Journal of Animal Science, 88, 3409-3418.

Rivero, M. A., Ramirez, J. A., Vazquez, J. M., Gil, F., Ramirez, G. \& Arencibia, A. 2005. Normal anatomical imaging of the thorax in three dogs: computed tomography and macroscopic cross sections with vascular injection. Anatomia, Histologia, Embryologia, 34, 215-219.

Roberts, N., Cruz-Orive, L. M., Reid, N. M. K., Brodie, D. A., Bourne, M. \& Edwards, R. H. T. 1993. Unbiased estimation of human body composition by the Cavalieri method using magnetic resonance imaging. Journal of Microscopy, 171, 239-253.

Sainz, R. D. 2000. Avaliação de carcaças e cortes comerciais de carne caprina e ovina. Simpósio Internacional sobre Caprinos e Ovinos de Corte, 1, 237-250.

Santos, C. L., Pérez, J. R. O., Muniz, J. A., Geraseev, L. C. \& Siqueira, E. R. 2001. Desenvolvimento relativo dos tecidos ósseo, muscular e adiposo dos cortes da carcaça de cordeiros Santa Inês. Revista Braseleira de Zootecnia, 30, 487-492.

Sañudo, C., Macie, E. S., Olleta, J. L., Villarroel, M., Panea, B. \& Albertí, P. 2004. The effects of slaughter weight, breed type and ageing time on beef meat quality using two different texture devices. Meat Science, 66, 925-932. 
Sehested, E. 1986. In-vivo prediction of lamb carcass composition by computerized tomography. Department of Animal Science. Agricultural University of Norway.

Sen, A. R., Santra, A. \& Karim, S. A. 2004. Carcass yield, composition and meat quality attributes of sheep and goat under semiarid conditions. Meat Science, 66, 757-763.

Silva, L. F., Pires, C. C. \& Silva, J. H. S. 2000. Crescimento de cordeiros abatidos com diferentes pesos. 1. Osso, músculo e gordura da carcaça e de seus cortes. Ciência Rural, 30, 671-675.

Silva Sobrinho, A. G., Purchas, R. W., Kadim, I. T. \& Yamamoto, S. M. 2005. Características de qualidade da carne de ovinos de diferentes genótipos e idades ao abate. Revista Brasileira de Zootecnia, 34, 1070-1078.

Silva, S. R., Gomes, M. J., Dias, A. S., Gil, L. F. \& Azevedo, J. T. 2005. Estimation in vivo of the body and carcass chemical composition of growing lambs by real-time ultrasonography. Journal of Animal Science, 83, 350-357.

Sonesson, A. K., De Greef, K. H. \& Meuwissen, T. H. E. 1998. Genetic parameters and trends of meat quality, carcass composition and performance traits in two selected lines of Large White pigs. Livestock Production Science, 57, 23-32.

Standal, N. 1984. Establishment of CT facility for farm animals. In vivo measurement of body composition in meat animals. Elsevier Applied Science Publishers, London, 1, 43-51.

Suguisawa, L. 2002. Ultra-sonografia para predição das características e composição da carcaça de bovinos. Universidade de São Paulo.

Susin, I. \& Mendes, C. Q. 2007. Confinamento de cordeiros: uma visão crítica. Simpósio de caprinos e ovinos, 2, 123-155.

Tarouco, J. U., Lobato, J. F. P., Tarouco, A. K. \& Massia, G. S. 2005. Relação entre medidas ultra-sônicas e espessura de gordura subcutânea ou área de olho de lombo na carcaça em bovinos de corte. Revista Brasileira de Zootecnia, 34, 2074-2084.

Viana, J. G. A. 2008. Panorama geral da ovinocultura no mundo e no Brasil. Revista Ovinos, 4, 1-9.

Wegener, O. H. 1992. Whole-body computed tomography. Blackwell Scientific Publications, Cambridge.

\section{Article History:}

Received 4 November 2016

Accepted 12 December 2016

Available on line 29 December 2016

License information: This is an open-access article distributed under the terms of the Creative Commons Attribution License 4.0, which permits unrestricted use, distribution, and reproduction in any medium, provided the original work is properly cited. 